\title{
Electric quadrupole moment and the tensor magnetic polarizability of twisted electrons and a potential for their measurements
}

\author{
Alexander J. Silenko ${ }^{1,2,3}, \exists$ Pengming Zhang ${ }^{1,4}, \oplus$ and Liping Zou ${ }^{1,4}$ \\ ${ }^{1}$ Institute of Modern Physics, Chinese Academy of Sciences, Lanzhou 730000, China \\ ${ }^{2}$ Bogoliubov Laboratory of Theoretical Physics, \\ Joint Institute for Nuclear Research, Dubna 141980, Russia \\ ${ }^{3}$ Research Institute for Nuclear Problems, \\ Belarusian State University, Minsk 220030, Belarus and \\ ${ }^{4}$ University of Chinese Academy of Sciences, \\ Yuquanlu 19A, Beijing 100049, China
}

(Dated: February 20, 2019)

\begin{abstract}
For a twisted (vortex) Dirac particle in nonuniform electric and magnetic fields, the relativistic Foldy-Wouthuysen Hamiltonian is derived including high order terms describing new effects. The result obtained shows for the first time that a twisted spin- $1 / 2$ particle possesses a tensor magnetic polarizability and a measurable (spectroscopic) electric quadrupole moment. We have calculated the former parameter and have evaluated the latter one for a twisted electron. The tensor magnetic polarizability of the twisted electron can be measured in a magnetic storage ring because a beam with an initial orbital tensor polarization acquires a horizontal orbital vector polarization. The electric quadrupole moment is rather large and strongly influences the dynamics of the intrinsic orbital angular momentum. Three different methods of its measurements, freezing the intrinsic orbital angular momentum and two resonance methods, are proposed. The existence of the quadrupole moment of twisted electrons can lead to practical applications.
\end{abstract}

\footnotetext{
*Electronic address: alsilenko@mail.ru

${ }^{\dagger}$ Electronic address: zhpm@impcas.ac.cn

${ }^{\ddagger}$ Electronic address: zoulp@impcas.ac.cn
} 
The discovery of twisted (vortex) electron beams [1] whose existence was predicted in Ref. [2] has shown that particles can carry an intrinsic orbital angular momentum (OAM). Since twisted electrons possess large magnetic moments, this discovery opens new possibilities in the electron microscopy and investigations of magnetic phenomena (see Refs. [3-10] and references therein). Twisted electron beams with large intrinsic OAMs (up to 1000ћ) have been recently obtained [11]. Basic properties of free twisted beams have been considered in Refs. [12, 13]. The dynamics of the intrinsic OAM in external magnetic and electric fields has been studied in Refs. [2, 14-18]. The general relativistic description of the classical and quantum dynamics of the intrinsic OAM in arbitrary electric and magnetic fields has been discussed in Refs. [19] and [20], respectively. In Ref. [20], the relativistic quantum dynamics of twisted (vortex) electrons has been constructed in the Schrödinger form on the basis of the relativistic Foldy-Wouthuysen (FW) transformation [21-23]. In the present work, an application of the approach used in Ref. [20] allows us to obtain new fundamental properties of twisted electron beams. We demonstrate for the first time that a twisted spin1/2 particle can possess a large measurable (spectroscopic) electric quadrupole moment (EQM) and a tensor magnetic polarizability (TMP). We calculate these new fundamental parameters (caused by the intrinsic OAM) for a twisted electron and develop methods for their measurements.

While a twisted electron is a single pointlike particle described by the standard Dirac equation, its wave function has a nontrivial spatial structure (see the reviews [3, 4]). A particle with an intrinsic OAM is characterized by nontrivial solutions of the Dirac and Schrödinger equations. Such solutions are coherent superpositions of partial plane waves with different momenta [3, 44, 24]].

The relativistic FW transformation (see Refs. 21 23] and references therein) being the relativistic generalization of the original method [25] can be applied to obtain the Schrödinger form of the relativistic quantum mechanics. The exact relativistic Hamiltonian in the FW representation (the FW Hamiltonian) for a twisted or a untwisted Dirac particle $(q=2)$ in a static (in general, nonuniform) magnetic field has been first obtained in Ref. [26] and is given by $(\hbar=1, c=1)[21,26-28]$

$$
\mathcal{H}_{F W}=\beta \sqrt{m^{2}+\boldsymbol{\pi}^{2}-e \boldsymbol{\Sigma} \cdot \boldsymbol{B}},
$$

where $\boldsymbol{\pi}=\boldsymbol{p}-e \boldsymbol{A}$ is the kinetic momentum, $\boldsymbol{B}$ is the magnetic induction, and $\beta$ and $\boldsymbol{\Sigma}$ are 
the Dirac matrices. This Hamiltonian acts on the bispinor $\Psi_{F W}=\left(\begin{array}{l}\phi \\ 0\end{array}\right)$.

A twisted electron is a charged centroid [2, 3]. Needed derivations are similar to those in Ref. [20]. However, second-order terms in $B$ should be calculated. We can suppose that the de Broglie wavelength, $\hbar / p$, is much smaller than the characteristic size of the nonuniformity region of the external field. Summing over partial waves with different momentum directions brings the operator $\boldsymbol{\pi}^{2}$ to the form [20]

$$
\boldsymbol{\pi}^{2}=\boldsymbol{\pi}^{\prime 2}+\mathfrak{p}^{2}-\frac{e}{2}[\boldsymbol{L} \cdot \boldsymbol{B}(\boldsymbol{R})+\boldsymbol{B}(\boldsymbol{R}) \cdot \boldsymbol{L}]+\frac{e^{2}}{4}[\boldsymbol{B}(\boldsymbol{R}) \times \mathfrak{r}]^{2},
$$

where $\boldsymbol{R}$ and $\boldsymbol{\pi}^{\prime}$ are the center-of-charge radius vector and the kinetic momentum of the centroid as a whole, $\mathfrak{r}=\boldsymbol{r}-\boldsymbol{R}$ and $\mathfrak{p}=-i \hbar \partial /(\partial \mathfrak{r})$ are internal canonical variables, and $\boldsymbol{L} \equiv \mathfrak{r} \times \mathfrak{p}$ is the intrinsic OAM [20]. The straightforward extraction of the square root [29] brings Eq. (11) into the form

$$
\begin{aligned}
\mathcal{H}_{F W}= & \beta \epsilon-\beta \frac{e}{4}\left(\frac{1}{\epsilon} \boldsymbol{\Lambda} \cdot \boldsymbol{B}(\boldsymbol{R})+\boldsymbol{B}(\boldsymbol{R}) \cdot \boldsymbol{\Lambda} \frac{1}{\epsilon}\right) \\
& +\beta \frac{e^{2}}{16}\left(\left\{\frac{1}{\epsilon},[\boldsymbol{B}(\boldsymbol{R}) \times \mathfrak{r}]^{2}\right\}-\left\{\frac{1}{\epsilon^{3}},[\boldsymbol{B}(\boldsymbol{R})]^{2}\right\}\right) \\
& -\beta \frac{e^{2}}{16}\left(\frac{1}{\epsilon^{3}}[\boldsymbol{L} \cdot \boldsymbol{B}(\boldsymbol{R})+\boldsymbol{B}(\boldsymbol{R}) \cdot \boldsymbol{L}](\boldsymbol{\Sigma} \cdot \boldsymbol{B}(\boldsymbol{R}))\right. \\
& \left.+(\boldsymbol{\Sigma} \cdot \boldsymbol{B}(\boldsymbol{R}))[\boldsymbol{L} \cdot \boldsymbol{B}(\boldsymbol{R})+\boldsymbol{B}(\boldsymbol{R}) \cdot \boldsymbol{L}] \frac{1}{\epsilon^{3}}\right) \\
& -\beta \frac{e^{2}}{64}\left\{\frac{1}{\epsilon^{3}},[\boldsymbol{L} \cdot \boldsymbol{B}(\boldsymbol{R})+\boldsymbol{B}(\boldsymbol{R}) \cdot \boldsymbol{L}]^{2}\right\}, \\
\epsilon= & \sqrt{m^{2}+\boldsymbol{\pi}^{2}+\mathfrak{p}^{2}} . \quad \boldsymbol{\Lambda}=\boldsymbol{L}+\boldsymbol{\Sigma} .
\end{aligned}
$$

In Eq. (2), all terms proportional to $1 / \epsilon^{3}$ were not previously taken into account. The curly brackets $\{\ldots, \ldots\}$ denote anticommutators. The additional terms appearing in the second order expansion of the square root in Eq. (11) in a power series in $1 / \epsilon$ are quadratic or bilinear in $\boldsymbol{B}$. Usually, $\epsilon^{2} \sim m^{2}=4.41 \times 10^{9} e \cdot \mathrm{T}, L \sim 100, B \sim 1 \mathrm{~T}$, and these terms are approximately 7 orders less than the main OAM-dependent term. Therefore, our previous results and conclusions [20] are correct, while the additional OAM-dependent terms define new physical effects. The second to last term in the FW Hamiltonian (2) characterizes the spin - intrinsic OAM coupling in the magnetic field and describes the additional spin precession caused by the intrinsic OAM. Reversing $\boldsymbol{L}$ changes the sign of the OAM-dependent correction to the spin precession frequency. The existence of the spin 
- intrinsic OAM coupling has been previously established in Refs. [3, 4, 14, 15]. The last term describing the tensor interaction of the intrinsic OAM with the magnetic field is similar to the corresponding spin tensor interaction. The operator of the latter interaction has the form $W=-\beta_{T}(\boldsymbol{S} \cdot \boldsymbol{B})^{2}$, where $\beta_{T}$ is the TMP defined in the particle rest frame and $\boldsymbol{S}$ is the spin matrix $\left[\boldsymbol{S}^{2}=s(s+1)\right]$. Thus, the TMP caused by the intrinsic OAM is given by

$$
W=-\beta \beta_{T}(\boldsymbol{L} \cdot \boldsymbol{B})^{2}, \quad \beta_{T}=\frac{e^{2} \hbar^{2}}{8 m^{3}}=5.25 \times 10^{4} \mathrm{fm}^{3} .
$$

The TMP of the twisted electron is much larger than TMPs of particles and nuclei conditioned by the spin interactions. In particular, for pointlike $\mathrm{W}^{ \pm}$bosons $\beta_{T}^{(W)} \sim 10^{-11} \mathrm{fm}^{3}$ [30, 31]. For the deuteron, the theoretical estimation is $\beta_{T}=0.195 \mathrm{fm}^{3}$ [32]. In addition, the tensor interaction of the twisted electron is proportional to $L^{2} \sim 10^{4}-10^{6}$.

The operator $\boldsymbol{L}$ commutes with $\mathfrak{p}^{2}$. The noncommutation of this operator with $[\boldsymbol{B}(\boldsymbol{R}) \times$ $\mathfrak{r}]^{2}$ does not lead to any important effects because the expectation values of the nonzero commutators $\left[L_{i},(\boldsymbol{B}(\boldsymbol{R}) \times \mathfrak{r})^{2}\right]\left(i=x, y\right.$ when $\left.\boldsymbol{B}(\boldsymbol{R})=B \boldsymbol{e}_{z}\right)$ are equal to zero.

Thus, the evolution of the intrinsic OAM does not reduce to its precession. The same assertion has been made in Refs. [3, 4, 15, 17]. However, Eq. (2) shows the existence of a new interaction caused by the TMP of the twisted Dirac particle. The corresponding relativistic classical equation has the same form as Eq. (11) (except for the spin term). Moreover, a consideration of a twisted centroid leads to the classical equation similar to Eq. (2). The tensor electric and magnetic polarizabilities caused by the spin interactions are common properties of nuclei with the spin $s \geq 1$. Besides this, the exact FW Hamiltonians for pointlike spin-1/2 and spin-1 particles (with $g=2$ ) in a uniform magnetic field (see Eq. (11) and Refs. [30, 31]) are almost identical (the only difference is the form of the spin matrices). The existence of the TMP for the pointlike spin-1 particles additionally substantiates its existence for the twisted Dirac ones. We also mention that the threecomponent spin operator $s=\hbar \Sigma / 2$ and the OAM operator $\boldsymbol{L}$ are defined in the particle rest frame and in the lab frame, respectively.

While the TMP of the twisted electron is large as compared with that of the deuteron, its measurement is a difficult experimental task. The TMP leads to very small shifts of energy levels and cannot be determined using the magnetic-resonance method. While $\left(B_{\|} \cos \omega t\right)^{2}=$ $B_{\|}^{2}(1+\cos 2 \omega t) / 2$, an oscillating horizontal $(\|)$ magnetic field $\boldsymbol{B}_{\|}$which frequency is half that of transitions between the Landau levels cannot stimulate any resonance. This occurs 
because the operator $\left(\boldsymbol{B}_{\|} \cdot \boldsymbol{L}\right)^{2}$ (unlike the operator $\boldsymbol{B}_{\|} \cdot \boldsymbol{L}$ ) does not mix neighboring energy levels. The same situation takes place for the tensor polarizabilities caused by the spin. In particular, the operators $\left(\boldsymbol{E}_{\|} \cdot \boldsymbol{S}\right)^{2},\left(\boldsymbol{B}_{\|} \cdot \boldsymbol{S}\right)^{2}$ mix only the levels $s_{z}= \pm 1$ for spin-1 particles [33 36].

The best possibilities to measure the TMP of the twisted electron are provided by the effects found in Ref. [37] and investigated in detail in Refs. [34, 38]. If the TMP is caused by the spin interactions, it produces a spin rotation with two frequencies instead of one, beating with a frequency proportional to $\beta_{T}$, and transitions between vector and tensor polarizations [37, 38]. Following Ref. [34], we propose to use a tensor-polarized twisted electron beam in a magnetic storage ring. In this case, the TMP is the only reason of the appearance of a horizontal orbital vector polarization of the beam. This polarization grows almost linearly in time [29, 34]. The horizontal component of the intrinsic OAM rotates with the Larmor frequency. The experiment can be performed in an electron storage ring or in a Penning trap. It needs a high beam coherency. To reach such a coherency, some methods developed for the electric-dipole-moment experiment [39] can be applied.

We also consider OAM-dependent interactions proportional to field derivatives. Contrary to the pointlike electron, the twisted electron (centroid) has a highly anisotropic spatial structure. Such an object possesses the EQM, while this property has not been previously mentioned. The Laguerre-Gaussian wave function describing a wave beam [3, 4] does not allow a rigorous determination of the electron density shape in the centroid rest frame $\left(p_{z} \rightarrow\right.$ 0 ). It is natural to assume that this is a strongly oblate (pancake shaped) spheroid. Twisted electron states in a uniform magnetic field [3, 14] always have such a shape. In this case, the intrinsic EQM of the centroid is given by $(e=-|e|)$

$$
Q_{0}=-e<\mathfrak{r}^{2}>=-e \frac{\int \varrho(\mathfrak{r}) \mathfrak{r}^{3} d \mathfrak{r}}{\int \varrho(\mathfrak{r}) \mathfrak{r} d \mathfrak{r}},
$$

where $\varrho(\mathfrak{r})=\left|\Psi_{F W}\right|^{2}$ is the electron density and $\mathfrak{r}$ is the radial coordinate of the cylindrical coordinate system introduced relative to the center of charge of the centroid. In the nonrelativistic approximation, the square of the $\mathrm{FW}$ wave function reduces to the square of the corresponding Schrödinger wave function.

The interaction of an extended charged particle with a static electric field is defined by the operator

$$
W=-\frac{1}{2}[\boldsymbol{d} \cdot \boldsymbol{E}(\boldsymbol{R})+\boldsymbol{E}(\boldsymbol{R}) \cdot \boldsymbol{d}]-\frac{1}{12}\left\{Q_{i j}, \frac{\partial E_{i}(\boldsymbol{R})}{\partial X_{j}}\right\},
$$


where $\boldsymbol{d}$ and $Q_{i j}$ are the operators of the electric dipole moment (see Ref. [20]) and the EQM, respectively. In the centroid rest frame, the EQM interacts only with an electric field. The EQM operator of the twisted electron averaged on states with the specific total angular momentum $\boldsymbol{j}=\boldsymbol{L}+\boldsymbol{s}$ is defined in the centroid rest frame and is given by

$$
Q_{i j}=\frac{3 Q_{s}}{2 j(2 j-1)}\left[\left\{j_{i}, j_{j}\right\}-\frac{2}{3} \delta_{i j} j(j+1)\right],
$$

where $Q_{s}$ is the spectroscopic EQM. Its connection with the intrinsic EQM has the form [40, 41]

$$
Q_{s}=\frac{3 K^{2}-j(j+1)}{(j+1)(2 j+3)} Q_{0}
$$

where $K$ is the projection of the total angular momentum onto the symmetry axis of the particle. In the case at hand, $j \gg 1$ and $Q_{s} \approx Q_{0}$.

For the Landau problem, eigenfunctions of the nonrelativistic and relativistic Hamiltonians coincide [20]. For twisted and untwisted electrons, the mean square of the radial cylindrical coordinate of the pointlike electron is given by [14]

$$
<r^{2}>=\frac{w_{m}^{2}}{2}\left(2 n+\left|l_{z}\right|+1\right), \quad w_{m}=2 \sqrt{\frac{\hbar}{|e B|}} .
$$

Here $w_{m}$ is the beam waist [3, 14] and $\boldsymbol{l}$ is the sum of the intrinsic and extrinsic OAMs. When $B=1 \mathrm{~T}, w_{m}=5.1 \times 10^{-8} \mathrm{~m}$.

The diameter of the vortex beam depending on the OAM has been determined in Refs. [8, 42]. It is about $10 \mathrm{~nm}$ when the topological charge is $\mathfrak{m}=50\left(L_{z}=\mathfrak{m} \hbar\right)$ and is proportional to $\mathfrak{m}[42]$.

The spin also contributes to the EQM of the twisted electron. An orbital motion of the magnetic moment of a spinning particle leads to the appearance of an electric current quadrupole moment (ECQM) [43]:

$$
Q_{i j}^{(\text {curr })}=-\frac{1}{2 \epsilon}\left[3 L_{i} \mu_{j}+3 L_{j} \mu_{i}-2 \delta_{i j}(\boldsymbol{L} \cdot \boldsymbol{\mu})\right], \quad \boldsymbol{\mu}=\frac{e \boldsymbol{s}}{\epsilon} .
$$

Its appearance results in a small correction to the spin precession frequency. The sign of this correction is defined by the sign of $L_{z}$. The ECQMs are comparatively small $\left(Q^{(\text {curr })} \sim\right.$ $\left.\mathrm{eL} / \mathrm{m}^{2}\right)$. The correction to the spin precession frequency is of the following order:

$$
\Delta \Omega \sim L\left|\frac{\partial E_{i}}{\partial X_{j}}\right|_{\max } \times 10^{-10} \mathrm{~s}^{-1}
$$


where the maximum gradient of the electric field is given in units of $\mathrm{V} / \mathrm{m}^{2}$. The ECQMs cause a spin - intrinsic OAM coupling.

Let us analyze the potential for measuring the EQM of twisted electrons in storage rings when $L \gg 1$ and spin effects are neglected. It is convenient to determine the dynamics of $L_{z}$. Relativistic effects in interactions of EQMs of spinning particles with electric and magnetic fields have been described in Refs. [44 [46]. Since polarization effects conditioned by the spin and intrinsic OAM are similar [29], we can use the results [44-46] with substituting $\boldsymbol{L}$ for $\boldsymbol{S}$ and taking into account that $L>>1$ and $\boldsymbol{V} \cdot \boldsymbol{E}=0(\boldsymbol{V}$ is the centroid velocity operator). The quadrupole interaction in the lab frame reads $\left(\widetilde{\boldsymbol{\beta}}=\boldsymbol{V} / c=\widetilde{\beta} \boldsymbol{e}_{\phi}\right)$

$$
\begin{aligned}
W & =-\frac{Q_{s}}{4 L^{2}}\left[(\boldsymbol{L} \cdot \nabla)-\frac{\gamma}{\gamma+1}(\boldsymbol{L} \cdot \widetilde{\boldsymbol{\beta}})(\widetilde{\boldsymbol{\beta}} \cdot \nabla)\right](\boldsymbol{L} \cdot \mathcal{E}), \\
\mathcal{E} & =\boldsymbol{E}(\boldsymbol{R})+\frac{1}{2}[\widetilde{\boldsymbol{\beta}} \times \boldsymbol{B}(\boldsymbol{R})-\boldsymbol{B}(\boldsymbol{R}) \times \widetilde{\boldsymbol{\beta}}],
\end{aligned}
$$

where $\mathcal{E}=\mathcal{E}(\boldsymbol{R})$ is the quasielectric field. All fields are defined in the lab frame. The noncommutativity of operators is neglected. The intrinsic OAM presented in Eq. (10) should not be confused with the extrinsic OAM. The operator $W$ should be added to the Hamiltonian (2). The use of a nonuniform magnetic field for focusing may be preferable.

The interaction operator $W$ contains the terms proportional to $\partial \mathcal{E}_{z} / \partial Z$ and $\partial \mathcal{E}_{r} / \partial R(R$ is the radial coordinate of the cylindrical coordinate system). The former term commuting with $L_{z}$ can be disregarded. The effect of the latter term is defined by

$$
W=-\frac{Q_{s}}{4 L^{2}} L_{r}^{2} \frac{\partial \mathcal{E}_{r}}{\partial R} .
$$

The last term in Eq. (6) does not contribute to the interaction operator (11) because $\partial \mathcal{E}_{i} / \partial X_{i} \equiv \operatorname{div} \mathcal{E}=0$.

To determine the effect of $W$ on the dynamics of the intrinsic OAM, we can use the fact that the components of the spin and the OAM satisfy equivalent commutation relations. Therefore, the OAM polarization tensor $\left\{L_{i}, L_{j}\right\}$ rotates in external fields with the same angular velocity as the OAM (the spin polarization tensor possesses the equivalent property [47]). The dynamics of the intrinsic OAM is defined by the large term [20]

$$
\begin{aligned}
\frac{d \boldsymbol{L}}{d t} & =\frac{1}{2}(\boldsymbol{\Omega} \times \boldsymbol{L}-\boldsymbol{L} \times \boldsymbol{\Omega}), \\
\boldsymbol{\Omega} & =-\beta \frac{e}{4}\left\{\frac{1}{\epsilon}, \boldsymbol{B}(\boldsymbol{R})\right\}+\frac{e}{4}\left[\frac{1}{\epsilon^{2}} \boldsymbol{\pi}^{\prime} \times \boldsymbol{E}(\boldsymbol{R})-\boldsymbol{E}(\boldsymbol{R}) \times \boldsymbol{\pi}^{\prime} \frac{1}{\epsilon^{2}}\right]
\end{aligned}
$$


and by corrections to this term caused by the EQM. Other corrections are defined by Eqs. (2) and (3). In Eq. (12), $\Omega$ is the operator of the angular velocity of Larmor precession in external fields. The Larmor precession caused by the vertical magnetic field and by the radial electric one (if the latter field is also used) does not change $L_{z}$.

In Eqs. (21), (11), and (12), a noncommutativity of the operators of coordinate and intrinsic OAM can be ignored because the operators $\mathfrak{p}$ and $\boldsymbol{L}$ are defined only by the internal coordinates.

The change of $L_{z}$ caused by the quadrupole interaction (11) is observable only when the Larmor precession is eliminated. This can be done by freezing the intrinsic OAM [19] in a specific combination of vertical magnetic and radial electric fields equalizing the angular velocities of the beam rotation and the intrinsic-OAM one $(\boldsymbol{\omega}=\boldsymbol{\Omega})$. An angle between the intrinsic OAM and the momentum remains unchanged. A similar method of freezing the spin [48] may be applied in electric-dipole-moment experiments. Freezing the intrinsic OAM takes place when [19]

$$
\boldsymbol{B}_{0}=\left(\frac{2}{\widetilde{\beta}^{2}}-1\right) \widetilde{\boldsymbol{\beta}} \times \boldsymbol{E}, \quad \boldsymbol{\omega}=-\frac{e \boldsymbol{B}_{0}}{m c \gamma\left(\gamma^{2}+1\right)} .
$$

If magnetic focusing is used, the magnetic field $\boldsymbol{B}$ is nonuniform and $\boldsymbol{B}_{0}$ is an average magnetic field. The forces caused by the electric and magnetic fields are oppositely directed. Electrons move counterclockwise. The ring radius is defined by

$$
R_{0}=\frac{V}{\omega}=\left(\gamma^{2}+1\right) \sqrt{\gamma^{2}-1} \frac{m c^{2}}{|e| B_{0}} .
$$

The nonuniformity of $\boldsymbol{B}$ leads to a nonuniform electric field in the centroid rest frame and to a turn of the intrinsic OAM in the vertical plane. In this case,

$$
\frac{\partial B_{z}}{\partial R}=-\frac{n B_{0}}{R_{0}}, \quad 0<n<1
$$

where $n$ is the field index. In Eq. (11), $\mathcal{E}_{r}=\widetilde{\beta} B_{z}$ and $\partial \mathcal{E}_{r} / \partial R=-\widetilde{\beta} n B_{0} / R_{0}$.

The commutator of the total FW Hamiltonian (including $W$ ) with the OAM operator results in the following addition to the equation of motion:

$$
\frac{d L_{z}}{d t}=\frac{Q_{s} \widetilde{\beta} n B_{0}}{4 L^{2} R_{0}}\left\{L_{r}, L_{\phi}\right\}
$$

Therefore, a beam with an initial horizontal orbital polarization acquires a vertical orbital polarization (cf. the similar spin effect [33-38, 49]). The beam can be tensor-polarized 
(when two beams with opposite orbital polarizations are joined) or vector-polarized. Let $\psi$ be the angle defining the orbital polarization relative to the $\boldsymbol{e}_{r}$ and $\boldsymbol{e}_{\phi}$ axes. The azimuth $\psi=0$ characterizes the intrinsic OAM directed radially outward. The change of $L_{z}$ is maximum when the direction of the initial horizontal orbital polarization satisfies the condition $|\sin 2 \psi|=1$ (cf. Refs. [29, 33, 35]).

The effect of the EQM on the OAM dynamics is very strong and can be easily observed. When the beam energy is equal to $300 \mathrm{keV}$ and $R_{0}=0.5 \mathrm{~m}, \widetilde{\beta}=0.777, E=2.46 \mathrm{MV} / \mathrm{m}$, $B_{0}=0.0148 \mathrm{~T}, f=\omega /(2 \pi)=7.41 \times 10^{7} \mathrm{~Hz}$. When $L \sim 100$ and the OAM diameter is determined based on the data presented in Ref. [42], the quantity $Q_{s} /\left(|e| R_{0}\right) \sim 10^{-16}$ $\mathrm{m}$ is not negligible as compared with the reduced Compton wavelength of the electron $\lambda_{C}=\hbar /(m c)=3.86 \times 10^{-13} \mathrm{~m}$. The frequency of the cyclic evolution of the orbital polarization (cf. Refs. [33-35, 37, 38, 49]) is 5 orders of magnitude less than the cyclotron frequency $f$ and can be properly measured. The corresponding terms in the FW Hamiltonian also differ by 5 orders of magnitude. Therefore, the main intrinsic-OAM dynamics is correctly described by the equations obtained in Refs. [19, 20], while the new EQM-dependent effect is rather important.

There is a systematical error caused by the small vertical electric field and the corresponding radial magnetic field $<B_{r}>=-<E_{z}>/ \widetilde{\beta}$ leading to a vanishing average Lorentz force (cf. Ref. [48]). This systematical error originates from field misalignments. However, it seems to be small and can be eliminated in measurements at two values of the field index.

The EQM of the twisted electron can also be measured by the magnetic-resonance method. In this case, a constant electric field is not needed. The resonance effect is provided by a nonuniform field oscillating with the angular frequency $\Omega$. The resonance field vanishing in the center of the beam trajectory, $B_{z}=\mathfrak{B}\left(R-R_{0}\right) \cos (\Omega t+\varphi)$ or $E_{r}=\mathfrak{E}\left(R-R_{0}\right) \cos (\Omega t+\varphi)$, is preferable. It is well known that such a field is equivalent to two fields rotating with the angular velocities $\boldsymbol{\Omega}$ [see Eq. (12)] and $\boldsymbol{\Omega}$. The first of these creates the resonance effect. In the frame rotating with the angular velocity $\Omega$, the intrinsic-OAM dynamics is similar to that when the intrinsic OAM is frozen [29].

The magnetic-resonance method provides the less sensitivity than the method of freezing the intrinsic OAM because the resonance field usually covers a small part of the ring circumference. Nevertheless, the effect of the EQM on the intrinsic-OAM dynamics can be properly detected. Otherwise, the magnetic-resonance method allows one to apply a stronger mag- 
netic field, a smaller ring size, and, therefore, a lower number of twisted electrons. Another advantage of this method is a simpler experimental setup.

The third method of measuring the EQM of the twisted electron is based on a standard stimulation of resonance transitions by an oscillating longitudinal magnetic field. This method, unlike the previous one, is sensitive to the quadrupole splitting of Landau levels defined by Eq. (11). The splitting is caused by the focusing magnetic field creating a nonuniform electric field in the electron rest frame. Therefore, the quadrupole splitting is proportional to the field index $n$. The stimulating magnetic field can be conditioned by a usual rf cavity and is longitudinal because such a field does not affect the beam motion. The considered method is similar not only to the magnetic-resonance method but also to the nuclear-quadrupole-resonance one. The resonance frequencies defined by a quadrupole structure of the energy levels depend on $n$.

The three methods considered need an increase in the currently available beam intensity. However, similar experiments can be carried out with a single twisted electron in a Penning trap.

Large EQMs of twisted electrons rather strongly interact with nonuniform electric fields. We expect that the twisted electrons can be successfully used not only in investigations of magnetic properties (see Refs. [3 7, 9, 10] and references therein) but also for nanoscale measurements of nonuniform electric fields in matter.

In this Letter, we have calculated minor terms in the relativistic FW Hamiltonian describing a twisted Dirac particle in nonuniform electric and magnetic fields. The results presented by Eqs. (2) - (6) have shown for the first time that the twisted electron $(s=1 / 2)$ possesses a TMP and a spectroscopic EQM. We have calculated the former parameter and have evaluated the latter one. It is still generally accepted that only particles and nuclei with spin $s \geq 1$ are characterized by these parameters. The TMP of the twisted electron is several orders of magnitude bigger than that of the deuteron. It can be measured in a magnetic storage ring because a beam with the initial orbital tensor polarization acquires a horizontal orbital vector polarization. The EQM is rather large and strongly influences the dynamics of the intrinsic OAM. We propose three different methods of its measurements, freezing the intrinsic OAM and two resonance methods. We expect that the existence of the EQM of twisted electrons can find practical applications because the EQM interaction with nonuniform electric fields in matter depends on the intrinsic-OAM direction. All the con- 
sidered effects also take place for twisted positrons. Additional explanations are presented in the Supplemental Material [29].

This work was supported by the Belarusian Republican Foundation for Fundamental Research (Grant No. \$18D-002), by the National Natural Science Foundation of China (Grants No. 11575254 and No. 11805242), by the National Key Research and Development Program of China (No. 2016YFE0130800), and by the Heisenberg-Landau program of the German Federal Ministry of Education and Research (Bundesministerium für Bildung und Forschung). A. J. S. also acknowledges hospitality and support by the Institute of Modern Physics of the Chinese Academy of Sciences. The authors are grateful to I. P. Ivanov and O. V. Teryaev for helpful exchanges.

[1] M. Uchida, A. Tonomura, Generation of electron beams carrying orbital angular momentum, Nature (London) 464, 737 (2010); J. Verbeeck, H. Tian, P. Schattschneider, Production and application of electron vortex beams, Nature 467, 301 (2010).

[2] K. Bliokh, Y. Bliokh, S. Savel'ev, and F. Nori, Semiclassical Dynamics of Electron Wave Packet States with Phase Vortices, Phys. Rev. Lett. 99, 190404 (2007).

[3] K. Y. Bliokh, I. P. Ivanov, G. Guzzinati, L. Clark, R. Van Boxem, A. Béché, R. Juchtmans, M. A. Alonso, P. Schattschneider, F. Nori, and J. Verbeeck, Theory and applications of freeelectron vortex states, Phys. Rep. 690, 1 (2017).

[4] S. M. Lloyd, M. Babiker, G. Thirunavukkarasu, and J. Yuan, Electron vortices: Beams with orbital angular momentum, Rev. Mod. Phys. 89, 035004 (2017).

[5] S. M. Lloyd, M. Babiker, G. Thirunavukkarasu, and J. Yuan, Electromagnetic Vortex Fields, Spin, and Spin-Orbit Interactions in Electron Vortices, Phys. Rev. Lett. 109, 254801 (2012).

[6] J. Rusz, S. Bhowmick, M. Eriksson, N. Karlsson, Scattering of electron vortex beams on a magnetic crystal: Towards atomic-resolution magnetic measurements, Phys. Rev. B 89, 134428 (2014).

[7] A. Edström, A. Lubk, J. Rusz, Elastic scattering of electron vortex beams in magnetic matter, Phys. Rev. Lett. 116, 127203 (2016).

[8] A. Béché, R. Juchtmans, J. Verbeeck, Efficient creation of electron vortex beams for high resolution STEM imaging, Ultramicroscopy 178, 12 (2017). 
[9] V. Grillo, T. R. Harvey, F. Venturi, J. S. Pierce, R. Balboni, F. Bouchard, G. C. Gazzadi, S. Frabboni, A. H. Tavabi, Z. Li, R. E. Dunin-Borkowski, R. W. Boyd, B. J. McMorran, E. Karimi, Observation of nanoscale magnetic fields using twisted electron beams, Nat. Commun. 8, 689 (2017).

[10] B. J. McMorran, A. Agrawal, P. A. Ercius, V. Grillo, A. A. Herzing, T. R. Harvey, M. Linck and J. S. Pierce, Origins and demonstrations of electrons with orbital angular momentum, Phil. Trans. R. Soc. A 375, 20150434 (2017).

[11] V. Grillo, G. C. Gazzadi, E. Karimi, E. Mafakheri, R. W. Boyd, and S. Frabboni, Highly Efficient Electron Vortex Beams Generated by Nanofabricated Phase Holograms, Appl. Phys. Lett. 104, 043109 (2014); V. Grillo, G. C. Gazzadi, E. Mafakheri, S. Frabboni, E. Karimi, and R. W. Boyd, Holographic Generation of Highly Twisted Electron Beams, Phys. Rev. Lett. 114, 034801 (2015); E. Mafakheri, A. H. Tavabi, P.-H. Lu, R. Balboni, F. Venturi, C. Menozzi, G. C. Gazzadi, S. Frabboni, A. Sit, R. E. Dunin-Borkowski, E. Karimi, and V. Grillo, Realization of electron vortices with large orbital angular momentum using miniature holograms fabricated by electron beam lithography, Appl. Phys. Lett. 110, 093113 (2017); H. Larocque, I. Kaminer, V. Grillo, G. Leuchs, M. J. Padgett, R. W. Boyd, M. Segev, E. Karimi, 'Twisted' electrons, Contemp. Phys. 59, 126 (2018).

[12] I. Bialynicki-Birula and Z. Bialynicka-Birula, Relativistic electron wave packets carrying angular momentum, Phys. Rev. Lett. 118, 114801 (2017).

[13] S. M. Barnett, Relativistic electron vortices, Phys. Rev. Lett. 118, 114802 (2017).

[14] K. Y. Bliokh, P. Schattschneider, J. Verbeeck, F. Nori, Electron vortex beams in a magnetic field: A new twist on Landau levels and Aharonov-Bohm states, Phys. Rev. X 2, 041011 (2012).

[15] G. M. Gallatin, B. McMorran, Propagation of vortex electron wave functions in a magnetic field, Phys. Rev. A 86, 012701 (2012); C. R. Greenshields, R. L. Stamps, S. Franke-Arnold, S. M. Barnett, Is the angular momentum of an electron conserved in a uniform magnetic field? Phys. Rev. Lett. 113240404 (2014); D. Chowdhury, B. Basu, and P. Bandyopadhyay, Electron vortex beams in a magnetic field and spin filter, Phys. Rev. A 91, 033812 (2015); K. van Kruining, A. G. Hayrapetyan, and J. B. Götte, Nonuniform currents and spins of relativistic electron vortices in a magnetic field, Phys. Rev. Lett. 119, 030401 (2017); A. Rajabi and J. Berakdar, Relativistic electron vortex beams in a constant magnetic field, Phys. Rev. A 95, 
$063812(2017)$.

[16] C. Greenshields, R. L. Stamps, and S. Franke-Arnold, Vacuum Faraday effect for electrons, New J. Phys. 14, 103040 (2012).

[17] Y. D. Han, T. Choi, Classical understanding of electron vortex beams in a uniform magnetic field, Phys. Lett. A 381, 1335 (2017).

[18] M. Babiker, J. Yuan, and V. E. Lembessis, Electron vortex beams subject to static magnetic fields, Phys. Rev. A 91, 013806 (2015); J. Qiu, C. Ren, and Z. Zhang, Precisely measuring the orbital angular momentum of beams via weak measurement, Phys. Rev. A 93063841 (2016).

[19] A. J. Silenko, Pengming Zhang and Liping Zou, Manipulating Twisted Electron Beams, Phys. Rev. Lett. 119, 243903 (2017).

[20] A. J. Silenko, Pengming Zhang and Liping Zou, Relativistic quantum dynamics of twisted electron beams in arbitrary electric and magnetic fields, Phys. Rev. Lett. 121, 043202 (2018).

[21] A. J. Silenko, Foldy-Wouthuysen transformation for relativistic particles in external fields, J. Math. Phys. 44, 2952 (2003).

[22] A. J. Silenko, Classical limit of equations of the relativistic quantum mechanics in the FoldyWouthuysen representation, Pis'ma Zh. Fiz. Elem. Chast. Atom. Yadra 10, 144 (2013) [Phys. Part. Nucl. Lett. 10, 91 (2013)].

[23] A. J. Silenko, General method of the relativistic Foldy-Wouthuysen transformation and proof of validity of the Foldy-Wouthuysen Hamiltonian, Phys. Rev. A 91, 022103 (2015).

[24] I. P. Ivanov, Measuring the phase of the scattering amplitude with vortex beams, Phys. Rev. D 85, 076001 (2012); I. P. Ivanov, D. Seipt, A. Surzhykov, and S. Fritzsche, Elastic scattering of vortex electrons provides direct access to the Coulomb phase, Phys. Rev. D 94, 076001 (2016).

[25] L. L. Foldy, S. A. Wouthuysen, On the Dirac Theory of Spin 1/2 Particles and Its NonRelativistic Limit, Phys. Rev. 78, 29 (1950).

[26] K. M. Case, Some Generalizations of the Foldy-Wouthuysen Transformation, Phys. Rev. 95, 1323 (1954).

[27] W. Tsai, Energy eigenvalues for charged particles in a homogeneous magnetic field - an application of the Foldy-Wouthuysen transformation, Phys. Rev. D 7, 1945 (1973).

[28] A. J. Silenko, Connection between wave functions in the Dirac and Foldy-Wouthuysen representations, Phys. Part. Nucl. Lett. 5, 501 (2008). 
[29] See Supplemental Material for some details relating to the derivation of Eq. (2) and for the additional explanations of internal-OAM dynamics of twisted electrons.

[30] A. J. Silenko, High precision description and new properties of a spin-1 particle in a magnetic field, Phys. Rev. D 89, 121701(R) (2014).

[31] A. J. Silenko, Quantum-mechanical description of spin-1 particles with electric dipole moments, Phys. Rev. D 87, 073015 (2013).

[32] J.-W. Chen, H. W. Grießhammer, M. J. Savage, R. E Springer, The polarizability of the deuteron, Nucl. Phys. A 644, 221 (1998); X. Ji, Y. Li, Sum rules and spin-dependent polarizabilities of the deuteron in effective field theory, Phys. Lett. B 591, 76 (2004).

[33] A. J. Silenko, Tensor electric polarizability of the deuteron in storage-ring experiments, Phys. Rev. C 75, 014003 (2007).

[34] A. J. Silenko, Potential for measurement of the tensor magnetic polarizability of the deuteron in storage ring experiments, Phys. Rev. C 77, 021001(R) (2008).

[35] A. J. Silenko, Potential for measurement of the tensor polarizabilities of nuclei in storage rings by the frozen spin method, Phys. Rev. C 80, 044315 (2009).

[36] V. G. Baryshevsky and A. J. Silenko, Potential for the measurement of the tensor electric and magnetic polarizabilities of the deuteron in storage-ring experiments with polarized beams, J. Phys.: Conf. Ser. 295, 012034 (2011).

[37] V. G. Baryshevsky, A. A. Gurinovich, Spin rotation and birefringence effect for a particle in a high energy storage ring and measurement of the real part of the coherent elastic zeroangle scattering amplitude, electric and magnetic polarizabilities, arXiv: hep-ph/0506135 V.G. Baryshevsky, About influence of the deuteron electric and magnetic polarizabilities on measurement of the deuteron EDM in a storage ring, arXiv: hep-ph/0510158.

[38] V. G. Baryshevsky, Rotation of particle spin in a storage ring with a polarized beam and measurement of the particle EDM, tensor polarizability and elastic zero-angle scattering amplitude, J. Phys. G: Nucl. Part. Phys. 35, 035102 (2008).

[39] G. Guidoboni et al. (JEDI Collaboration), How to Reach a Thousand-Second in-Plane Polarization Lifetime with 0.97-GeV/c Deuterons in a Storage Ring, Phys. Rev. Lett. 117, 054801 (2016).

[40] A. J. Buchmann and E. M. Henley, Intrinsic quadrupole moment of the nucleon, Phys. Rev. C 63, 015202 (2000). 
[41] G. Neyens, Nuclear magnetic and quadrupole moments for nuclear structure research on exotic nuclei, Rep. Prog. Phys. 66, 633 (2003).

[42] K. Saitoh, Y. Hasegawa, N. Tanaka and M. Uchida, Production of electron vortex beams carrying large orbital angular momentum using spiral zone plates, J. Electron Microsc. 61, $171(2012)$.

[43] A. J. Silenko, Current electric quadrupole moments of atoms and nuclei, Sov. Phys. J. 34, 607 (1991); Electric current quadrupole moments of atoms and nuclei, J. Phys. B 25, 1661 (1992); Electric current multipole moments in classical electrodynamics, Prog. Theor. Phys. 101, 875 (1999).

[44] A. A. Pomeranskii and I. B. Khriplovich, Equations of motion of spinning relativistic perticle in external fields, J. Exp. Theor. Phys. 86, 839 (1998); A. A. Pomeransky and R. A. Sen'kov, Quadrupole interaction of relativistic quantum particle with external fields, Phys. Lett. B 468, 251 (1999).

[45] A. J. Silenko, Quadrupole and contact interaction of relativistic particles with the electric field of crystals, Phys. Atom. Nucl. 63, 2045 (2000).

[46] A. J. Silenko, The Motion of Particle Spin in a Nonuniform Electromagnetic Field, J. Exp. Theor. Phys. 96, 775 (2003); Analysis of wave equations for spin-1 particles interacting with an electromagnetic field, arXiv: hep-th/0404074

[47] A. J. Silenko, General dynamics of tensor polarization of particles and nuclei in external fields, J. Phys. G: Nucl. Part. Phys. 42, 075109 (2015).

[48] F. J. M. Farley, K. Jungmann, J. P. Miller, W. M. Morse, Y. F. Orlov, B. L. Roberts, Y. K. Semertzidis, A. Silenko, and E. J. Stephenson, New Method of Measuring Electric Dipole Moments in Storage Rings, Phys. Rev. Lett. 93, 052001 (2004).

[49] V. Baryshevsky and A. Shirvel, The deuteron (nuclei) birefringence effect in a matter and in an electric field and the searches for an EDM of a deuteron (nucleus) rotating in a storage ring, arXiv: hep-ph/0503214; V. G. Baryshevsky, Birefringence effect in the nuclear pseudoelectric field of matter and an external electric field for a deuteron (nucleus) rotating in a storage ring, arXiv: hep-ph/0504064.

[50] In Refs. [33-35], the initial spin tensor polarization characterizes a deuteron beam with the zero spin projection onto the fixed direction. In the present study, we consider the initial orbital tensor polarization which differs in sign and value from the above-mentioned polarization. 


\section{Supplemental Material to "Electric quadrupole moment and the tensor magnetic polarizability of twisted electrons and a potential for their measurements"}

In this Supplemental Material, we specify a derivation of Eq. (2) in our Letter and present some additional explanations for internal-OAM dynamics of twisted electrons caused by interactions bilinear in the internal OAM $\boldsymbol{L}$.

When some operators $\mathcal{A}$ and $\mathcal{B}$ do not commute, their double commutators can be neglected, and the operator $\mathcal{B}$ is comparatively small,

$$
\sqrt{\mathcal{A}+\mathcal{B}}=\frac{1}{2}\{\sqrt{\mathcal{A}(1+x)}\}=\frac{1}{2}\{\sqrt{\mathcal{A}}, \sqrt{1+x}\}=\frac{1}{2}\left\{\sqrt{\mathcal{A}},\left(1+\frac{x}{2}-\frac{x^{2}}{8}\right)\right\}
$$

where $x=\frac{1}{2}\left\{\mathcal{A}^{-1}, \mathcal{B}\right\}$. With the equivalent assumptions, the extraction of the square root allows us to pass from Eq. (1) to Eq. (2) in the Letter.

The explanations for internal-OAM dynamics of twisted electrons are based on previous studies of spin dynamics of deuterons caused by interactions bilinear in the spin [33-38, 47, 49]. Since these studies have been performed for deuterons, some of them [33 35] have used the fixed spin number $s=1$. Nevertheless, it is well-known that the use of the Pauli spin matrices describing spin-1/2 particles allow one to determine the spin rotation of particles with any spins. Similarly, the application of results obtained in Refs. [33 35] gives a right qualitative description of the spin dynamics of particles and nuclei with spins $s>1$ and the intrinsic-OAM dynamics. Therefore, the results obtained in Refs. [33 38, 49] with different quantum-mechanical and classical approaches are in the best compliance. Moreover, a correction needed for a description of the intrinsic-OAM dynamics usually reduces to the change $\boldsymbol{S} \rightarrow \boldsymbol{L}$ and an addition of the factor $2 L-1$.

There is a deep similarity between polarization effects conditioned by the intrinsic OAM $\boldsymbol{L}$ and the spin $\boldsymbol{S}$ caused by the equivalent commutation relations between the components of these operators:

$$
\left[L_{i}, L_{j}\right]=i e_{i j k} L_{k}, \quad\left[S_{i}, S_{j}\right]=i e_{i j k} S_{k},
$$

where the square brackets denote commutators. The polarization vectors and the polariza- 
tion tensors for the OAM and the spin are also equivalent:

$$
\begin{array}{ll}
P_{i}=\frac{<L_{i}>}{L}, & P_{i j}=\frac{3<L_{i} L_{j}+L_{j} L_{i}>-2 L(L+1) \delta_{i j}}{2 L(2 L-1)}, \\
P_{i}=\frac{<S_{i}>}{s}, & P_{i j}=\frac{3<S_{i} S_{j}+S_{j} S_{i}>-2 s(s+1) \delta_{i j}}{2 s(2 s-1)},
\end{array}
$$

where $P_{i j}=P_{j i}$ and $P_{\rho \rho}+P_{\phi \phi}+P_{z z}=1$. In the considered case, $i, j$ denote projections onto the axes of the cylindrical coordinate system. This similarity allows one to apply formulas defining the spin dynamics for a description of the intrinsic-OAM dynamics. Equation (S1) shows that results obtained in spin physics are useful for the description of dynamics of the intrinsic OAM.

The interaction Hamiltonian depending on the intrinsic OAM can be presented in the form

$$
\mathcal{H}=\boldsymbol{\Omega} \cdot \boldsymbol{L}+\frac{1}{2} \alpha_{i j}\left(L_{i} L_{j}+L_{j} L_{i}\right)
$$

where $\Omega$ is the angular velocity of the Larmor precession of the intrinsic OAM in the cylindrical coordinate system. The equation of the intrinsic-OAM motion has the form

$$
\frac{d L_{k}}{d t}=(\boldsymbol{\Omega} \times \boldsymbol{L})_{k}+\frac{1}{2} \alpha_{i j}\left(e_{k i l}\left\{L_{l}, L_{j}\right\}+e_{k j l}\left\{L_{l}, L_{j}\right\}\right) .
$$

This equation shows that the minor second term in Eq. (S3) defines the change of the orbital vector polarization depending on the orbital tensor polarization. This effect allows one to obtain an orbital vector polarization of an initially tensor-polarized and vectorunpolarized twisted beam (cf. the similar spin effects [33-35]). Such an initial polarization can be reached by mixing two twisted beams with antiparallel intrinsic OAMs. The second term in Eq. (S3) also influences the dynamics of the orbital vector polarization of an initially vector-polarized twisted beam.

An additional equation for $d P_{i j} /(d t)$ completes the system of equations defining the intrinsic-OAM dynamics. Such a system of equation has been constructed in Refs. [37, 38, 49] for a description of spin effects. When the effect of the second term in the interaction Hamiltonian (S2) on the dynamics of the tensor polarization can be neglected, the tensor polarization is constant in the frame rotating with the angular velocity $\Omega$. As a result, the first term in the interaction Hamiltonian conditions a rotation of the tensor polarization with this angular velocity [47]. The neglect of the influence of the second term on the dynamics of the tensor polarization allows one to consider only Eq. (S3 $)$. However, a change of this dynamics caused by the second term in Eq. (S2) can also be important. 
Components of the polarization vector and the polarization tensor of vector- and tensorpolarized twisted beams are defined as well as in the spin physics. When the initial twisted beam is vector-polarized and the direction of its orbital polarization is defined by the spherical angles $\theta$ and $\psi$,

$$
\begin{gathered}
P_{\rho}(0)=\sin \theta \cos \psi, \quad P_{\phi}(0)=\sin \theta \sin \psi, \quad P_{z}(0)=\cos \theta, \\
P_{\rho \rho}(0)=\frac{1}{2}\left(3 \sin ^{2} \theta \cos ^{2} \psi-1\right), \quad P_{\phi \phi}(0)=\frac{1}{2}\left(3 \sin ^{2} \theta \sin ^{2} \psi-1\right), \\
P_{z z}(0)=\frac{1}{2}\left(3 \cos ^{2} \theta \cos ^{2} \psi-1\right), \quad P_{\rho \phi}(0)=\frac{3}{4} \sin ^{2} \theta \sin (2 \psi), \\
P_{\rho z}(0)=\frac{3}{4} \sin (2 \theta) \cos \psi, \quad P_{\phi z}(0)=\frac{3}{4} \sin (2 \theta) \sin \psi .
\end{gathered}
$$

When the direction of an initial tensor polarization of a twisted beam obtained by mixing two beams with antiparallel intrinsic OAMs is defined by the spherical angles $\theta$ and $\psi$,

$$
\boldsymbol{P}(0)=0
$$

and the components of the polarization tensor are the same.

Equation (3) in our Letter is equivalent to the corresponding equation describing the spin-dependent tensor magnetic polarizability. The presence of the matrix $\beta$ in Eq. (3) is not important because the lower Foldy-Wouthuysen spinor is zero. As a result, the effects of the polarization vector rotation with two frequencies instead of one, beating with a frequency proportional to $\beta_{T}$, and transitions between vector and tensor polarizations [34, 35, 37, 38] also take place for twisted beams with an orbital polarization. All formulas obtained in these works remain applicable [50]. As follows from the results obtained in Refs. [34, 35], the above-mentioned vector-unpolarized twisted beam with the orbital tensor polarization defined by Eq. (S5) acquires the orbital vector polarization. When the uniform magnetic field is parallel to the $z$ axis,

$$
\begin{aligned}
\mathcal{H} & =\boldsymbol{\Omega} \cdot \boldsymbol{L}-\beta_{T} B^{2} L_{z}^{2}, \\
\frac{d L_{\rho}}{d t} & =(\boldsymbol{\Omega} \times \boldsymbol{L})_{\rho}+\beta_{T} B^{2}\left\{L_{\phi}, L_{z}\right\}, \\
\frac{d L_{\phi}}{d t} & =(\boldsymbol{\Omega} \times \boldsymbol{L})_{\phi}-\beta_{T} B^{2}\left\{L_{\rho}, L_{z}\right\} .
\end{aligned}
$$

Equations (S1) and (S5) show that the orbital tensor polarization should not be horizontal. Its optimal vertical direction is defined by the angle $\theta=\pi / 4$. The intrinsic-OAM dynamics is given by formulas obtained in Refs. [34, 35, 37, 38]. For a twisted particle with 
$L=1$ and the above-mentioned orbital tensor polarization, the general equation defining this dynamics has the form (see Refs. [34, 35])

$$
\begin{aligned}
P_{\rho}(t) & =-\frac{1}{2} \sin (2 \theta) \sin (\Omega t+\psi) \sin (b t), \\
P_{\phi}(t) & =\frac{1}{2} \sin (2 \theta) \cos (\Omega t+\psi) \sin (b t), \\
P_{z}(t) & =0, \quad b=-\beta_{T} B^{2} .
\end{aligned}
$$

The final vector polarization is horizontal and its absolute value increases. When $L>1$, Eq. (S8) is valid up to a constant factor. The electric quadrupole moment (EQM) of a particle affects the intrinsic-OAM dynamics only when the magnetic field is nonuniform.

The effect of the EQM of twisted electrons on the dynamics of the intrinsic OAM also needs some explanations. When the method of freezing the intrinsic OAM is used, the angular velocity of the Larmor precession is vanished and the intrinsic-OAM motion is defined by Eq. (16) in our Letter. A motion of the intrinsic OAM in the case of $\Omega=0$ is very similar to a spin behavior when the frozen spin method is used. The general description of this behavior is presented in Ref. [35]. We can use Eqs. (14), (15), and (19) from this article. In our case, with the same denotations as above, the dynamics of the vertical component of the orbital polarization is defined by

$$
P_{z}(t)=\cos (2 \mathcal{A} t) \cos \theta+\frac{1}{2} \sin ^{2} \theta \sin (2 \mathcal{A} t) \sin (2 \psi),
$$

when the initial beam of twisted electrons is vector-polarized and

$$
P_{z}(t)=\frac{1}{2} \sin ^{2} \theta \sin (2 \mathcal{A} t) \sin (2 \psi)
$$

when this beam is tensor-polarized. Here

$$
\mathcal{A}=-\frac{Q_{s} \widetilde{\beta} n B_{0}}{8 L^{2} R_{0}} .
$$

The tensor magnetic polarizability does not influence $P_{z}$ [35].

When the magnetic-resonance method of a measurement of the EQM is used, the intrinsic OAM rotates with the angular velocity of the Larmor precession, $\boldsymbol{\Omega}$. The angular frequency of the spin precession, $\Omega^{\prime}$, is defined by the well-known Thomas-Bargmann-Michel-Telegdi equation. Precedent studies of the spin-tensor effects fulfilled by different methods [33, 37, 38. have shown that the spin behavior perturbed by time-independent spin-vector and spintensor interactions is different. In the same static fields, the former and latter interactions 
lead to spin oscillations with the angular frequencies $\Omega^{\prime}$ and $2 \Omega^{\prime}$, respectively (see, e.g., Eq. (66) in Ref. [38] and Eq. (67) in Ref. [33]). To provide a resonance, the perturbing spintensor interaction should oscillate with the angular frequency $\omega \approx 2 \Omega^{\prime}[33,37,38]$. The same situation takes place for the intrinsic OAM. It can be similarly shown that the resonance frequency of the perturbing tensor interaction (10) is $\omega=2 \Omega$. The perturbing nonuniform quasielectric field is given by $\mathcal{E}_{r}(R, t)=\mathcal{E}_{r}(R) \cos (\omega t+\varphi)$. The quantity $\varphi$ is an oscillation phase at the moment of time $t=0$ when the initial beam polarization is defined by Eqs. (S4), (S5), and (S6). In the general case of an imperfect resonance, the dynamics of $P_{z}$ is defined by Eqs. (51) and (57) in Ref. [33] and is given by

$$
\begin{gathered}
P_{z}(t)=\left(1-\frac{2 \mathcal{A}^{2}}{\omega^{\prime 2}} \sin ^{2} \frac{\omega^{\prime} t}{2}\right) \cos \theta+\frac{\mathcal{A}}{\omega^{\prime}} \sin ^{2} \theta \sin \frac{\omega^{\prime} t}{2}\left[\frac{2 \Omega-\omega}{\omega^{\prime}} \sin \frac{\omega^{\prime} t}{2} \cos (2 \psi-\varphi)\right. \\
\left.+\cos \frac{\omega^{\prime} t}{2} \sin (2 \psi-\varphi)\right]
\end{gathered}
$$

and [50]

$$
P_{z}(t)=\frac{\mathcal{A}}{\omega^{\prime}} \sin ^{2} \theta \sin \frac{\omega^{\prime} t}{2}\left[\frac{2 \Omega-\omega}{\omega^{\prime}} \sin \frac{\omega^{\prime} t}{2} \cos (2 \psi-\varphi)+\cos \frac{\omega^{\prime} t}{2} \sin (2 \psi-\varphi)\right]
$$

when the initial beam is vector-polarized and tensor-polarized, respectively. Here

$$
\omega^{\prime}=\sqrt{(2 \Omega-\omega)^{2}+\mathcal{A}^{2}} .
$$

The resonance part of the tensor interaction is defined by

$$
\begin{aligned}
\mathcal{E}_{r}(R, t) & =\mathfrak{G}\left(R-R_{0}\right) \cos (\omega t+\varphi), \\
W & =-\frac{Q_{s} \mathfrak{G}}{4 L^{2}} \cos (\omega t+\varphi) L_{r}^{2}=2 \mathcal{A} \cos (\omega t+\varphi) L_{r}^{2}, \\
\mathcal{A} & =-\frac{Q_{s} \mathfrak{G}}{8 L^{2}} .
\end{aligned}
$$

The definitions of $\mathcal{A}$ in Eqs. (S11) and (S14) are different.

The third method of the measurement of the EQM is very similar to the nuclear quadrupole resonance method. However, the nuclear quadrupole resonance takes place in the rest frame of the moving electron. 